\title{
Biological Drug Neutralization
}

National Cancer Institute

\section{Source}

National Cancer Institute. Biological Drug Neutralization. NCI Thesaurus. Code C82903.

A physiological process in which body systems act on drugs, neutralizing their chemical effects. 